\title{
Multiple rotation assessment through isothetic fringes in speckle photography
}

\author{
Luciano Ángel, Myrian Tebaldi, and Néstor Bolognini
}

\begin{abstract}
The use of different pupils for storing each speckled image in speckle photography is employed to determine multiple in-plane rotations. The method consists of recording a four-exposure specklegram where the rotations are done between exposures. This specklegram is then optically processed in a whole field approach rendering isothetic fringes, which give detailed information about the multiple rotations. It is experimentally demonstrated that the proposed arrangement permits the depiction of six isothetics in order to measure either six different angles or three nonparallel components for two local general in-plane displacements. (C) 2007 Optical Society of America
\end{abstract}

OCIS codes: $120.0120,120.3180,120.3940,120.6160$.

\section{Introduction}

Speckle pattern can be used as a random carrier of information or for data storage. Another application is the use of speckle patterns for studying object displacements and distortions that arise in nondestructive testing of mechanical components currently addressed both in speckle interferometry and speckle photography. ${ }^{1-3}$ Speckle-based techniques could be employed in a wide range of applications, for instance, to measure the surface roughness, ${ }^{4}$ to determine mechanical and thermal microstrains, ${ }^{5}$ and to analyze vibrations.

Double-exposure speckle photography consists of recording a scattering object before and after deformation whose surface is illuminated by a laser beam. ${ }^{6,7}$ It is also possible to use white-light speckles to study object deformation. ${ }^{8}$ When the two laser speckle patterns are superimposed, no apparently discernible interference pattern results. However, if an optical Fourier transform and spatial filtering

L. Ángel is with the Grupo de Óptica Aplicada, Departamento de Ciencias Básicas, Universidad EAFIT, Medellín, Colombia. M. Tebaldi (myrianc@ciop.unlp.edu.ar) is with the Centro de Investigaciones Ópticas, CIOp (CONICET, CIC) and OPTIMO (Departamento Fisicomatemática, Facultad Ingeniería, Universidad Nacional de La Plata, P.O. Box 124 (1900), La Plata, Argentina. N. Bolognini is with the Facultad de Ciencias Exactas of the Universidad Nacional de La Plata.

Received 8 September 2006; revised 29 November 2006; accepted 14 December 2006; posted 19 December 2006 (Doc. ID 74797); published 23 April 2007.

0003-6935/07/142676-07\$15.00/0

(C) 2007 Optical Society of America technique are applied to the specklegram, fringes appear at the output plane. For analysis, a point-wise or a whole-field procedure can be applied to the doubleexposure speckle pattern. ${ }^{9}$

Let us consider the whole-field procedure. ${ }^{10}$ This procedure consists of two successive Fourier transforms of the specklegram to form an image of this input. In the first Fourier plane a filtering step is introduced, which allows us to depict fringes of equal components of displacements (i.e., isothetics) overlapped on the final image. The fringe period and the direction depend on the location of the filtering mask.

Note that generally when using a single aperture for recording a low signal-to-noise ratio is achieved, mainly because diffracted light at high frequencies is very low as a consequence of the light concentration in a tiny central region in the Fourier plane. This drawback results in isothetics of relatively low visibility. To overcome this limitation, several multiple-aperture approaches have been implemented, ${ }^{11,12}$ whose main advantage is the capability to concentrate diffracted light onto the high-frequency regions of the filtering plane. In this way, the amount of light available at the filtering location in the multiple-aperture case is higher than in the single-aperture case.

When recording, the same multiple-aperture pupil is currently employed in both exposures. In our proposal some key differences can be found. In previous work on the double-exposure specklegram by changing in different ways between exposures the apertures that constitute the pupil were analyzed. In Ref. 13 the use of different scale aperture pupils for image recording in speckle photography is proposed. The interferometric fringe visibility is theoretically and 
experimentally investigated. In Ref. 14 it is assumed that the double-aperture pupil is changed between exposures when locating, replacing, or removing a phase object in front of one of the apertures. In this arrangement the interferometric fringe behavior depends strongly on the phase object introduced in one aperture of the double-aperture system. As a consequence, this speckle interferometric technique allows us to characterize phase objects. Another possibility consists of using different multiple-aperture pupils that change between exposures in a multiple-exposure scheme. This particular scheme leads to storing different object statuses in the same specklegram and allows us to compare them. This method is presented in Ref. 15 but is restricted to analyzing multiple uniform in-plane displacements. This approach allows us to selectively combine the spectra components of determined speckled images into isolated spots in the Fourier plane. In this case, by considering different pupils for storing each speckled image, the ensemble average intensity in the Fourier plane is analytically derived, and the visibility of the fringes is investigated. The theoretical predictions obtained in this paper become a relevant tool for designing the recording routine and the set of pupils.

In what we believe to be a new contribution we consider the case of multiple rotations. As mentioned above, to measure displacements the whole-field analysis procedure is implemented. A four-exposure scheme by using different pupils in each exposure is performed. Each pupil consists of three apertures where two apertures coincide exactly and the remaining aperture does not overlap at all when any pair of pupils is considered. We experimentally demonstrate that with this particular scheme it is possible to depict six isothetics to measure either six different angles or three nonparallel components for two local general in-plane displacements.

\section{Recording and Analyzing Procedures}

For recording the specklegrams, a conventional photographic procedure was implemented. Referring to Fig. 1(a), a random diffuser $R$ at the input plane is illuminated by using a collimated $\mathrm{He}-\mathrm{Ne}$ laser beam $(\lambda=632.8 \mathrm{~nm})$ and imaged onto a high-resolution holographic film (Agfa-Gevaert 8E75) located at the $X-Y$ plane. To form the image, a lens $\mathrm{L}$ of focal length $f$ was employed. Immediately in front of the lens L, a mask with multiple circular apertures, which constitutes the pupil $\mathbf{P}$ of the system, was located. The distances from the input plane to the lens and from the lens to the image plane were denoted as $Z_{0}$ and $Z_{C}$, respectively, producing a diffuser magnification $M=Z_{C} / Z_{0}$.

A speckled image of the diffuser is produced through each aperture, whose diameter determines the average speckle size. The speckles produced in the image plane are internally modulated by fringes because waves going through different apertures are coherent and interfere. The characteristics of fringes that modulate individual speckles are determined by the relative positions of the apertures on the pupil. ${ }^{16}$ Spe- (a)

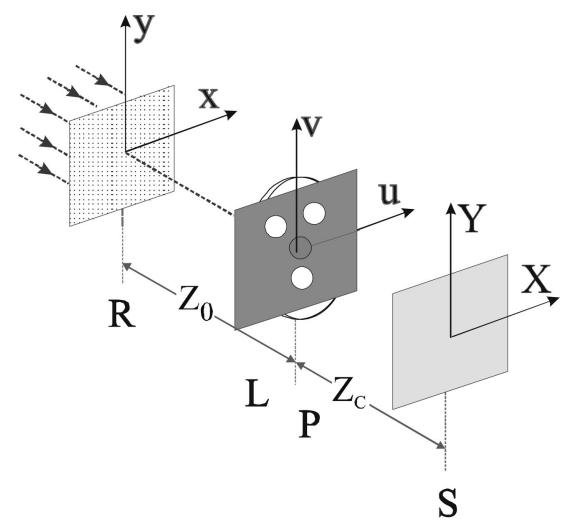

(b)

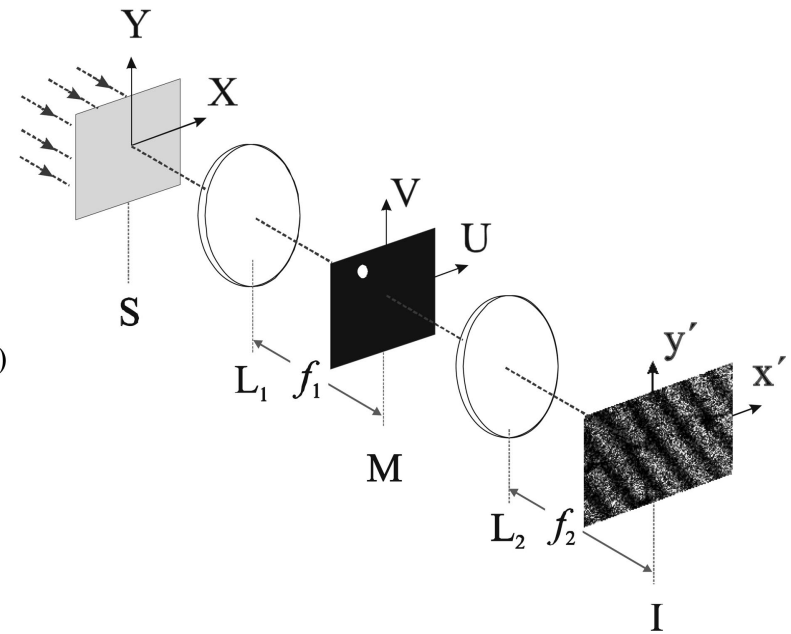

Fig. 1. Experimental arrangements for (a) recording step (R, random diffuser; L, lens; P, pupil mask; S, specklegram) and (b) analysis step (S, specklegram; $L_{1}$ and $L_{2}$, lenses; M, filtering mask; I, isothetics plane).

cifically, the intensity distribution that characterizes the internal modulation of speckles can be understood in terms of the incoherent superposition of all the fringe systems independently generated by the distinct pairs of apertures in the pupil.

The pupil $\mathbf{P}$ can be defined as

$$
P(u, v)=\sum_{n=1}^{N} a_{n}(u, v),
$$

where $a_{n}(u, v)$ represents the amplitude transmission function corresponding to the $n$th aperture. Each function $a_{n}(u, v)$ equals zero outside the respective apertures and equals unity inside them. Because the apertures are separated from each other, it follows that $a_{n}(u, v) a_{m}(u, v) \equiv 0$ for $n \neq m$. By considering circular apertures, let $\left(u_{n}, v_{n}\right)$ be the center of the $n$th generic aperture. Then each pair of apertures $a_{n}(u, v)$ and $a_{m}(u, v)$ can be associated with an elementary cosine system of fringes $f_{n m}$, whose direction is perpendicular to the line joining the center of the apertures and whose average period is $\Lambda_{n m}=\lambda Z_{C} / d_{n m}$, where $d_{n m}=\sqrt{\left(u_{m}-u_{n}\right)^{2}+\left(v_{m}-v_{n}\right)^{2}}$.

In our proposal, a multiple-exposure scheme by using multiple-aperture pupils is implemented. In- 
deed, for each exposure a different pupil is employed. The pupil associated with the $k$ th exposure is represented as $\mathbf{P}^{k}(u, v)(k=1,2, \ldots Q)$. As in Ref. 15, it is assumed that for any pair of pupils $\mathbf{P}^{k}(u, v)$ and $\mathbf{P}^{l}(u, v)(k \neq l)$, some of their apertures coincide exactly (common apertures), and the remaining apertures do not overlap at all (noncommon apertures). Then the whole recording procedure results in a transmittance function (the specklegram) proportional to the incoherent superposition of the modulated speckle intensity distributions corresponding to the diffuser images successively formed through the pupils. A linear response of the recording medium is assumed.

For analyzing local displacements, a whole-field procedure was implemented. By referring to Fig. 1(b), the specklegram at the $X-Y$ plane is illuminated by a coherent plane wave of wavelength $\lambda$. Afterward, two successive Fourier transforms of the specklegram are achieved by using lenses $L_{1}$ and $L_{2}$ of focal lengths $f_{1}$ and $f_{2}$, respectively. As in the conventional approaches, a filtering step is introduced in the UV plane to depict the equal components of displacements fringes (i.e., isothetics) onto the final image (in the $x^{\prime} y^{\prime}$ plane). To fully understand the filtering effect over the recorded image spectra and the subsequent aspect of the isothetic fringes in the output plane, an additional discussion about the Fourier plane information distribution must be done in relation to the characteristics of the pupil apertures.

Let $A^{k}(x, y)$ and $\mathbf{P}^{k}(u, v)$ represent the input complex amplitude and the pupil associated with the $k$ th exposure, respectively. The field amplitude $G(U, V)$ in the filtering $U-V$ plane can be expressed as the overlapping of the Fourier-transformed waves, each one denoted as $G^{k}(U, V)$ representing the complex amplitude associated with an individual exposure:

$$
G(U, V)=\sum_{k=1}^{Q} G^{k}(U, V)
$$

$G^{k}(U, V)$ is the autocorrelation given by

$$
G^{k}(U, V)=\left\{S^{k} \otimes\left(S^{k}\right)^{*}\right\}(U, V),
$$

where

$$
\begin{aligned}
S^{k}(\chi, \varsigma)= & P^{k}\left(-\chi \frac{Z_{C}}{f_{1}},-\varsigma \frac{Z_{C}}{f_{1}}\right) \\
& \times T^{k}\left(-\chi \frac{M}{f_{1}},-\varsigma \frac{M}{f_{1}}\right) \\
T^{k}\left(-\chi \frac{M}{f_{1}},-\varsigma \frac{M}{f_{1}}\right)= & \int A^{k}(x, y) \\
& \times \exp \left(i \frac{2 \pi}{\lambda} \frac{M}{f_{1}}(\chi x+\varsigma y)\right) \mathrm{d} x \mathrm{~d} y .
\end{aligned}
$$

According to Eqs. (2)-(4), each pupil limits the spectral content of each encoded image that contributes to the amplitude field in the filtering $U-V$ plane. In this plane, the spectral components of the input $A^{k}(x, y)$, associated with the $k$ th exposure, are concentrated in the regions where

$$
\left\{\mathbf{P}^{k}\left(-\chi \frac{Z_{C}}{f_{1}},-\varsigma \frac{Z_{C}}{f_{1}}\right) \otimes \mathbf{P}^{k}\left(-\chi \frac{Z_{C}}{f_{1}},-\varsigma \frac{Z_{C}}{f_{1}}\right)\right\}(U, V) \neq 0 .
$$

As a consequence of using multiple-aperture pupils, the spectral content of each image is gathered into the diffraction spots associated with the respective pupil. ${ }^{17}$ Then as introduced in Eq. (2), the whole spectral content consists of the overlapping of the spectra of the images that were recorded. These spectra can be selectively superimposed in the $U-V$ plane in accordance with the aperture arrangements that were employed.

In our approach, for any pair of multiple-aperture pupils $\mathbf{P}^{k}(u, v)$ and $\mathbf{P}^{l}(u, v)$ in the recording scheme, there is one pair of apertures in one pupil that coincides exactly with one pair of apertures in the other pupil; meanwhile the remaining apertures do not overlap at all. As a consequence, when comparing the speckled images corresponding to any pair of pupils, among the system of fringes modulating speckles, we can find one system that is similar in both patterns because of the pair of apertures the two pupils have in common. Indeed, when simultaneously Fourier transforming the stored images, these elementary fringe systems associated with a given pair of common apertures in the respective pupils generate the same pair of diffracted spots in the filtering plane. Then concerning the diffracted pattern, the loci of one spot pair associated with $\mathbf{P}^{k}$ coincide with the loci of one spot pair corresponding to $\mathbf{P}^{l}$. In those spots the spectra of both the $k$ th and $l$ th images are overlapped. The design of the pupil set for recording guarantees that each spot contains the spectra of only two images, as will be illustrated in detail in the next section. Then by properly filtering the light that impinges onto different diffraction spots, it is possible to selectively isolate the recorded information of all possible pairs of images. As a consequence, isothetic fringes associated with each spot depict the displacement components for a corresponding pair of images.

\section{Experimental Results}

To demonstrate the results of the previous section, experiments were addressed by implementing a fourexposure recording scheme. In Fig. 2(a), the four multiple-aperture pupils we actually employed are depicted. As can be observed, each pupil consists of three apertures, and for any pair of pupils $\mathbf{P}^{k}$ and $\mathbf{P}^{l}$, only two apertures coincide exactly and the remaining aperture does not overlap at all. In Fig. 2(b), the respective diffraction patterns are displayed. Note that any pair of diffraction patterns exhibits two com- 
(a)
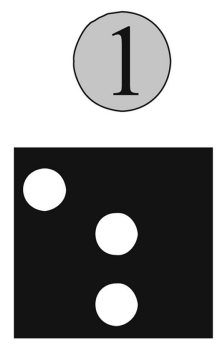

(b)

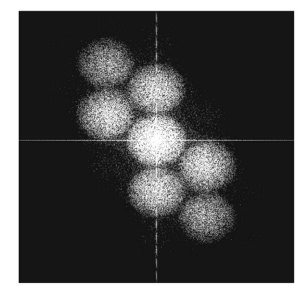

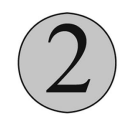
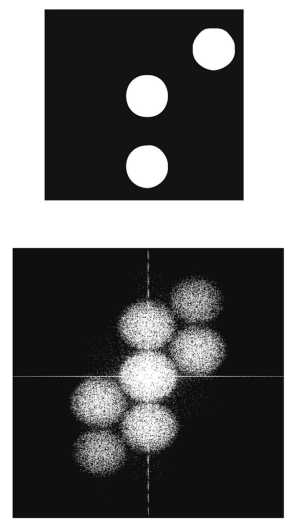
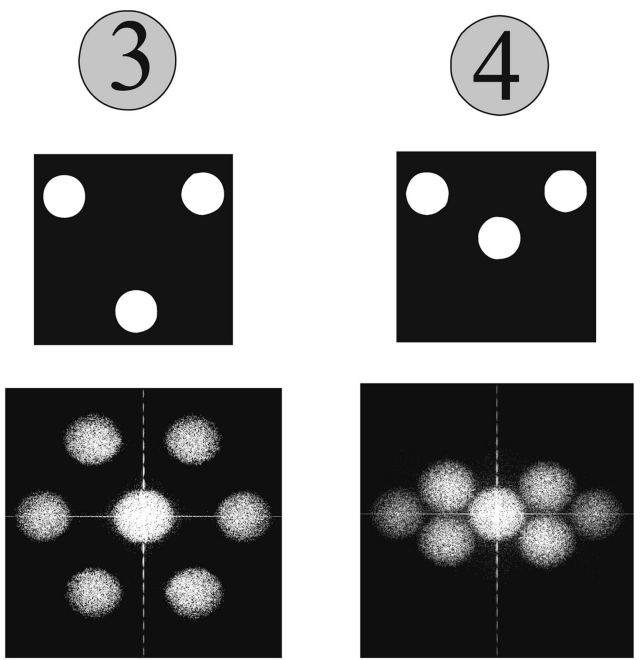

(c)

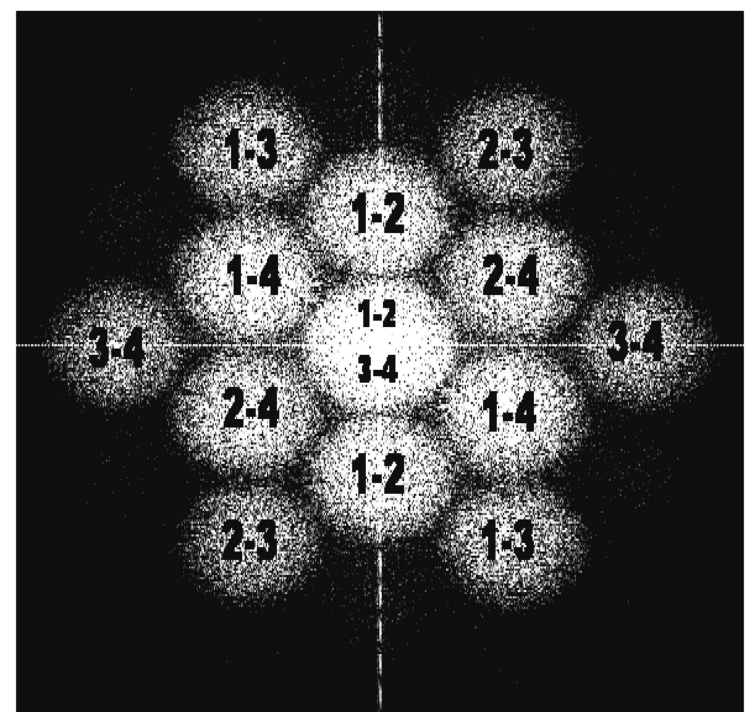

Fig. 2. (a) Multiple-aperture pupils indicated by (1), (2), (3), and (4). (b) Diffraction patterns corresponding to each pupil. (c) Diffraction patterns corresponding to a four-exposure specklegram. In each spot the numbers indicate which images (pupils) contribute to this spot.

mon diffracted spots while the others do not coincide despite the zero order.

The specklegram analysis procedure was conducted by using the setup schematized in Fig. 1(b). As mentioned in the previous section, the specklegram was coherently illuminated and the transmitted light was Fourier transformed in the $U-V$ plane by the lens $\mathrm{L}_{1}$ of focal length $f_{1}$. Afterward, the signal was filtered out by a single hole mask, and the light going through the hole was Fourier transformed in the $x^{\prime}-y^{\prime}$ plane by using a lens $\mathrm{L}_{2}$ of focal length $f_{2}$. Then in the output plane, a diffuser image with magnification $f_{2} / f_{1}$ was formed.

In the experiments we carried out, local displacements were introduced by in-plane rotation of the diffuser between exposures. Let us consider that the diffuser is rotated an angle $\theta \ll 1$. It follows that the magnitude of the diffuser displacement $|\vec{a}|$ at a distance $r$ from the rotation axis is given by $|\vec{a}|=$ $r \theta$. Then in the specklegram plane the speckle rela- tive displacement at the corresponding distance $R=$ $\left(Z_{C} / Z_{0}\right) r$ from the rotation axis is $|\vec{A}|=\left(Z_{C}|\vec{a}| / Z_{0}\right.$ $=R \theta$.

When analyzing the multiple-exposure specklegram, the four diffraction patterns superimpose onto the $U-V$ plane. In Fig. 2(c), the resulting diffraction pattern is displayed. Note that each diffraction spot contains information of only two images corresponding to two different diffuser positions. The two numbers in each spot refer to the images whose spectral information coincides there. It should be emphasized that by considering the whole diffraction pattern, the gathering of the spectral information for all possible image pairs is achieved. Therefore it is possible through the filtering step to selectively recover, in the $x^{\prime} y^{\prime}$ plane, the respective relative local displacements between any pair of diffuser positions. For filtering, a mask with a $1 \mathrm{~mm}$ diameter circular hole was employed. This mask was sequentially centered in each 


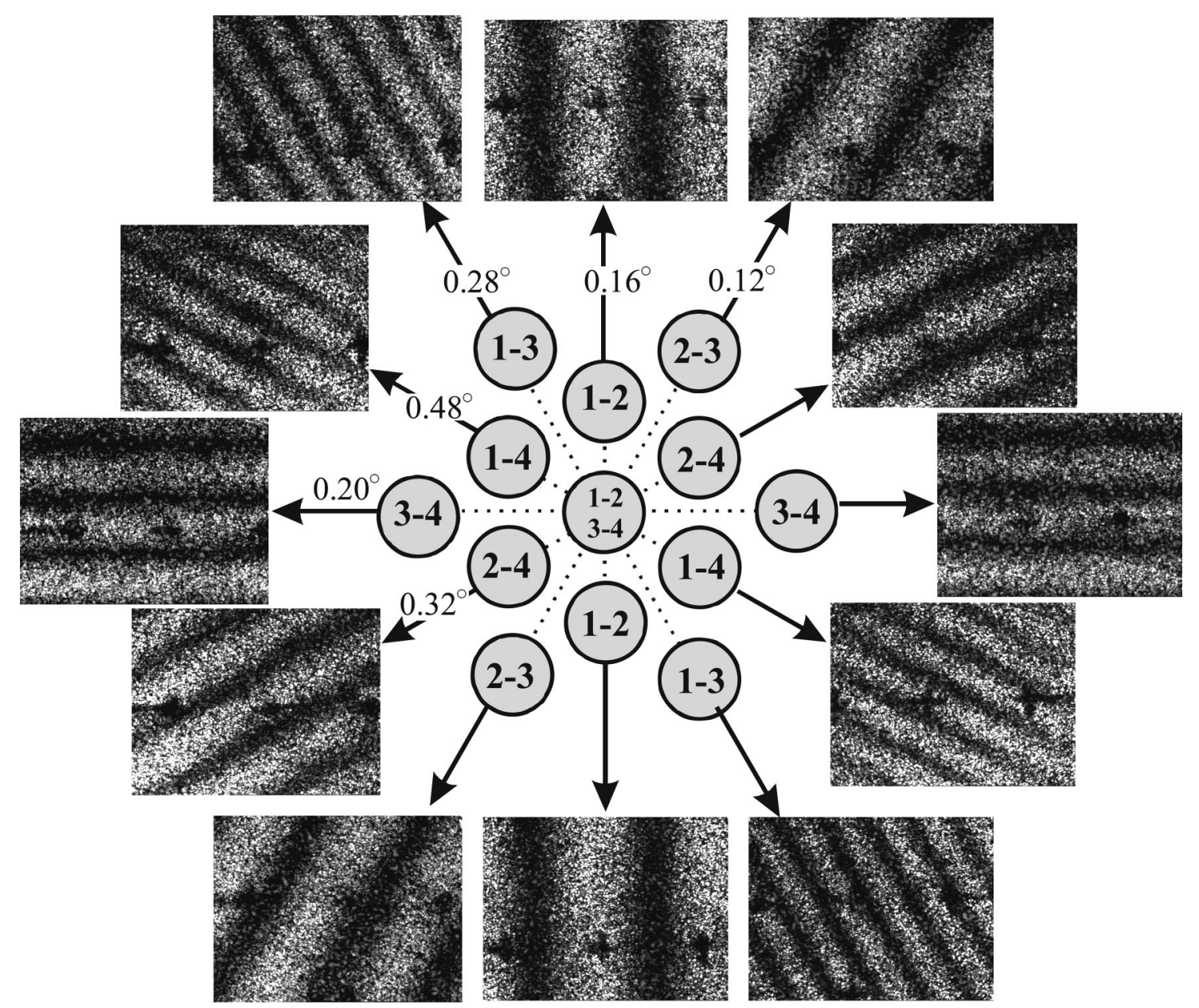

Fig. 3. Four-exposure diffraction pattern scheme. The isothetic fringes obtained by filtering out each spot are displayed. In each spot, the angle $\theta$ in which the diffuser was rotated is indicated.

spot. In Fig. 3 the isothetic fringes associated with the respective spots are presented. As expected, the isothetic direction coincides with the line joining the center of the respective spot and the optical axis.

Isothetics represent a family of equidistant fringes with cosine profile, which are perpendicular to the displacement vector $\vec{A}$, and the fringe period is $\lambda f_{2} /|\vec{A}|$. Moreover, if the position of the filtering aperture is indicated by the vector $\vec{T}$ in the $(U, V)$ plane, then the isothetic fringes will represent the loci of the points of equal displacement components along the perpendicular to the vector $\vec{T}$. Indeed, the bright fringes are given by $\vec{A} \vec{T}=n \lambda f_{2}$, where $n=0, \pm 1$, $\pm 2 \ldots$ Note that as the modulus $|\vec{T}|$ increases, the sensitivity of the technique increases proportionally. ${ }^{9}$

As it was mentioned, the filtering mask was located at the center of the diffracted spots. Then, for all the inner spots, the distance from the spot center to the zero order is $T_{1}=f_{1} d_{1} / Z_{C}$, where $d_{1}$ is the distance between the nearest aperture centers in the pupils. Similarly for all the outer spots, the distance from the spot center to the zero order is $T_{2}=f_{1} d_{2} / Z_{C}$, where $d_{2}$ is the distance between the farthest aperture centers in the pupils. This implies that the modulus

$$
|\vec{T}|= \begin{cases}T_{1} & \text { for inner spots } \\ T_{2} & \text { for outer spots }\end{cases}
$$

In our case, $d_{1}=16.0 \mathrm{~mm}$, and $d_{2}=\sqrt{3} d_{1}=$ $26.5 \mathrm{~mm}$. It is apparent that, according to the pupils' geometry, when an outer spot is filtered out, the sensitivity is $\sqrt{3}$ times the sensitivity when an inner spot is involved.

As introduced above, the isothetic fringes of Fig. 3 correspond to a four-exposure specklegram by an inplane rotation of the diffuser. The experiment was carried on in which the diffuser was rotated an angle: $\theta_{12}=0.16^{\circ}$ between the first and the second exposures, $\theta_{23}=0.12^{\circ}$ between the second and the third exposures, and $\theta_{34}=0.20^{\circ}$ between the last two exposures. Then the other relative angles we analyze are $\theta_{12}+\theta_{23}=\theta_{13}=0.28^{\circ}, \theta_{12}+\theta_{23}+\theta_{34}=\theta_{14}$ $=0.48^{\circ}$, and $\theta_{23}+\theta_{34}=\theta_{24}=0.32^{\circ}$. To verify the validity of our proposal, these angle values were compared with the values determined through the isothetic fringes. 

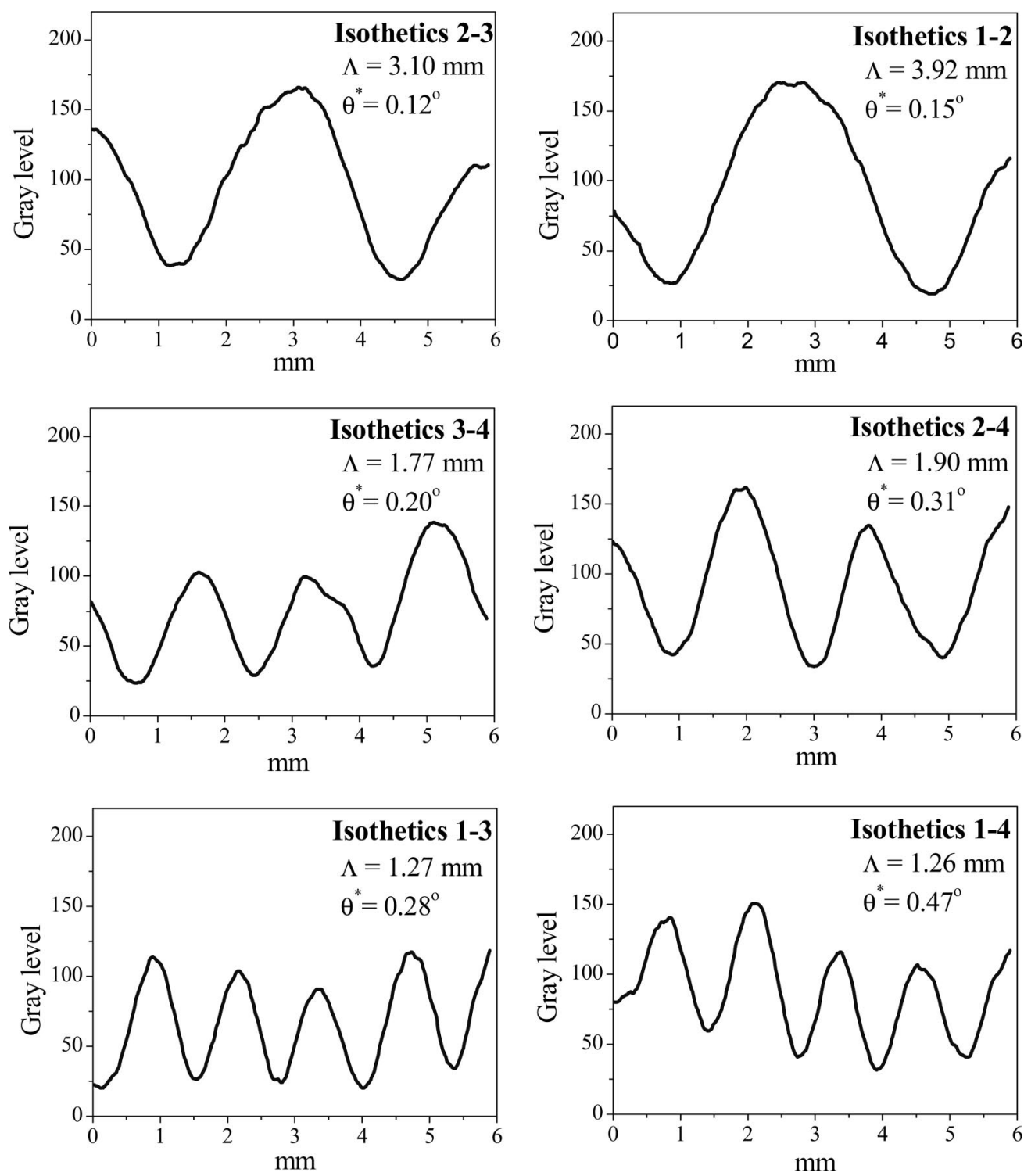

Fig. 4. Intensity profiles along a direction perpendicular to each isothetic fringe pattern. The corresponding diffracted spot, the isothetic fringe period, and the measured rotation $\theta^{*}$ angle are indicated in each case.

To measure these angles, the proper diffraction spot for filtering must be selected. In terms of the isothetics period $\Lambda$, the diffuser rotation angle $\theta$ between exposures could be calculated by using the expression

$\theta^{*}=\left\{\begin{array}{ll}\frac{\lambda f_{2}}{T_{1}} \frac{1}{\Lambda} & \text { when filtering at an inner spot } \\ \frac{\lambda f_{2}}{T_{2}} \frac{1}{\Lambda} & \text { when filtering at an outer spot }\end{array}\right.$.

In Fig. 4, the respective isothetics intensity profiles along a direction perpendicular to the fringes are shown. For each pair of equivalent isothetics only one profile is presented. Each profile includes the corresponding fringe period and the predicted angle $\theta^{*}$ by using Eq. (8), whose values coincide up to $0.01^{\circ}$ with the actual ones. The actual average speckle size at the isothetics plane was approximately $40 \mu \mathrm{m}$. Note that the ratio between the fringe period and the average speckle size is large enough (between 30 and 100), thereby allowing the achievement of high visibility isothetics.

\section{Conclusions}

The proposed technique stands for an extension of the conventional speckle photography to measure multiple local in-plane displacements. The technique is based on a multiple-exposure scheme by using different multiple-aperture pupils for recording. Each exposure corresponds to a particular input diffuser status associated with a given arrangement of pupil apertures. A comparison between all possible recorded images is possible, including nonconsecutive images. Note that the technique is particularly useful to asses discrete object status. In case a continuous object evolution needs to be evaluated, the status 
number to be characterized is limited by the pupil aperture scheme, the recording media response, and the time interval to change the pupil.

The input surface experiences local changes between exposures, and all various local displacements between images can be determined. To illustrate the main features of the technique, some experiments by in-plane rotation of the diffuser around the optical axis between exposures were conducted. In this case, only local displacement components perpendicular to the line joining the point of concern and the rotation axis are relevant. It implies that displacements can be assessed by measuring the respective rotation angle. However, the technique also enables us to make more general local displacement measurements by determining three nonparallel components. In particular, the proposed scheme is capable of depicting six isothetics to measure either six different angles or three nonparallel components for two local general in-plane displacements. Moreover, for each of the general displacements of concern, three equal sensitivity isothetics can be obtained.

The accuracy and precision of the proposed speckle photography extension is the same as in conventional schemes.

This research was performed under grants CONICET 5995 (Argentina), CICPBA (Argentina), ANCYT PICT 12564 (Argentina), Facultad de Ingeniería, Universidad Nacional de La Plata 11/I105 (Argentina), and Universidad EAFIT (Colombia). L. Angel acknowledges the financial support under the TWAS-UNESCO Associateship Scheme at Centers of Excellence in the South.

\section{References}

1. J. C. Dainty, ed., Laser Speckle and Related Phenomena (Springer-Verlag, 1975).

2. R. K. Erf, Speckle Metrology (Academic, 1978).
3. R. Sirohi, ed., Speckle Metrology (Marcel Dekker, 1993).

4. C. J. Tay, S. L. Toh, H. M. Shang, and J. Zhang, "Whole-field determination of surface roughness by speckle correlation," Appl. Opt. 34, 2324-2335 (1995).

5. F. P. Chiang, J. Adachi, R. Anastasi, and J. Beaty, "Subjective laser speckle method and its application to solid mechanics problems," Opt. Eng. 21, 379-390 (1982).

6. J. M. Burch and M. J. Tokarski, "Production of multiple beam fringes from photographic scatters," Opt. Acta 15, 101-111 (1968).

7. E. Archbold, J. M. Burch, and A. E. Ennos, "Recording of in-plane surface displacement by double-exposure speckle photography," Opt. Acta 17, 883-898 (1970).

8. F. P. Chiang and A. Asundi, "Perspective effect in the whitelight speckle method," Appl. Opt. 21, 1708-1710 (1982).

9. F. D. Chiang and R. P. Khetan, "Strain analysis by one-beam laser speckle interferometry. I. Single aperture method," Appl. Opt. 15, 2205-2215 (1976).

10. F. P. Chiang and C. J. Lin, "Stress analysis of in-plane vibration of 2D structures by a laser speckle method," Appl. Opt. 19, 2705-2707 (1980).

11. D. Duffy, "Moiré gauging of in-plane displacement using double aperture imagining," Appl. Opt. 11, 1778-1781 (1972).

12. F. D. Chiang and R. P. Khetan, "Strain analysis by one-beam laser speckle interferometry 2: Multiaperture method," Appl. Opt. 18, 2175-2186 (1979).

13. L. Angel, M. Tebaldi, M. Trivi, and N. Bolognini, "Fringe visibility analysis with different scale apertures in speckle photography," J. Mod. Opt. 43, 1749-1765 (2001).

14. M. Tebaldi, L. Angel, M. Trivi, and N. Bolognini, "Phase-object detection by use of double-exposure fringe modulated speckle patterns," J. Opt. Soc. Am. A 20, 116-129 (2003).

15. L. Angel, M. Tebaldi, N. Bolognini, and M. Trivi, "Speckle photography with different pupils in a multiple exposure scheme," J. Opt. Soc. Am. A 17, 107-119 (2000).

16. L. Angel, M. Tebaldi, M. Trivi, and N. Bolognini, "Properties of speckle patterns generated through multiaperture pupils," Opt. Commun. 192, 37-47 (2001).

17. L. Angel Toro, M. Tebaldi, M. Trivi, and N. Bolognini, "Optical operations based on speckle modulation by using a photorefractive crystal," Opt. Commun. 168, 55-64 (1999). 